\title{
Ischemia induces different levels of hypoxia inducible factor-1 a protein expression in interneurons and pyramidal neurons
}

Prabhu Ramamoorthy and Honglian Shi

\begin{abstract}
Introduction: Pyramidal (glutamatergic) neurons and interneurons are morphologically and functionally well defined in the central nervous system. Although it is known that glutamatergic neurons undergo immediate cell death whereas interneurons are insensitive or survive longer during cerebral ischemia, the protection mechanisms responsible for this interneuronal survival are not well understood. Hypoxia inducible factor-1 (HIF-1) plays an important role in protecting neurons from hypoxic/ischemic insults. Here, we studied the expression of HIF-1a, the regulatable subunit of HIF-1, in the different neuronal phenotypes under in vitro and in vivo ischemia.
\end{abstract}

Results: In a primary cortical culture, HIF-1a expression was observed in neuronal somata after hypoxia (1\% oxygen) in the presence of 5 or $25 \mathrm{mM}$ glucose but not under normoxia (21\% oxygen). Interestingly, only certain MAP2-positive neurons containing round somata (interneuron-like morphology) co-localized with HIF-1a staining. Other neurons such as pyramidal-like neurons showed no expression of HIF-1a under either normoxia or hypoxia. The HIF-1a positive neurons were GAD65/67 positive, confirming that they were interneuron-type cells. The HIF-1a expressing GAD65/67-positive neurons also possessed high levels of glutathione. We further demonstrated that ischemia induced significant HIF-1a expression in interneurons but not in pyramidal neurons in a rat model of middle cerebral artery occlusion.

Conclusion: These results suggest that HIF-1a protein expression induced by ischemia is neuron-type specific and that this specificity may be related to the intracellular level of glutathione (GSH).

\section{Introduction}

Neurons can be classified into three major groups, pyramidal neurons responsible for glutamate release, interneurons with round cell bodies responsible for $\gamma$-aminobutyric acid (GABA) release, and spiny interneurons with small cell bodies that can release both glutamate and GABA. Interestingly, ischemia-mediated vulnerability differs among neuronal subpopulations. Interneurons are resistant to ischemia in striatum, cortex, and hippocampus, whereas pyramidal neurons undergo immediate cell death under certain ischemic conditions [1-8]. However, the molecular mechanism for neuronal type-specific resistance to ischemia is not well understood.

Hypoxia inducible factor-1 (HIF-1) is a transcriptional factor that plays a critical role in cellular adaptation to

\footnotetext{
* Correspondence: hshi@ku.edu

Department of Pharmacology and Toxicology, School of Pharmacy, University of Kansas, 5064 Malott, Lawrence, KS 66045, USA
}

low oxygen levels. It is a heterodimer consisting of two subunits, $\alpha$ and $\beta$. HIF- $1 \beta$ is constitutively expressed; the oxygen level has no effect on its expression. The protein level of HIF-1 $\alpha$ is highly regulated by oxygen tension [9]. Thus, the activity of HIF-1 is primarily determined by the expression of the subunit HIF- $1 \alpha$ and not that of HIF-1 $\beta$. During hypoxia, HIF- $1 \alpha$ is stabilized, translocates to the nucleus, binds to HIF- $1 \beta$, and initiates transcription. HIF-1 plays an important role in neuroprotection against ischemia by upregulating various growth factors such as vascular endothelial growth factor and erythropoietin. It has been shown that HIF- $1 \alpha$ knockdown increases brain injury in a mouse model of transient focal cerebral ischemia [10]. Inhibition of proline hydroxylase (PHD), an enzyme that initiates the degradation of HIF- $1 \alpha$, protects against glutamateinduced damage in the rat hippocampus [11]. Moreover, it has been reported that HIF-1 $\alpha$ expression can vary in 
different cells. For example, its stability and degradation is regulated in a cell-type-specific manner in carcinoma cell lines [12]. The expression of HIF-1 $\alpha$ differs in hepatoma and primary endothelial cells due to different degradation mechanisms [13]. Degradation occurs mainly in the cytosol in HEPG2 cells and in both cytosol and nucleus in mouse brain endothelial cells [13]. Previous data from our laboratory demonstrate that HIF- $1 \alpha$ stability requires a reducing environment during ischemia and that increases in glutathione (GSH) levels stabilize HIF- $1 \alpha$ in cortical neurons [14], indicating that protein levels of HIF- $1 \alpha$ may vary among cells with different redox statuses.

We hypothesized that HIF-1 $\alpha$ was expressed differently in pyramidal neurons and interneurons during hypoxic conditions. To test this hypothesis, we studied and compared the cell-type-specific expression of HIF- $1 \alpha$ in pyramidal neurons and interneurons in a primary cortical neuronal culture exposed to hypoxia and an animal model of cerebral ischemia. We demonstrated that, under ischemic conditions, HIF- $1 \alpha$ expression was remarkably stable in interneurons when compared to pyramidal neurons. HIF-1 $\alpha$ stability in interneurons was consistent with an increase in intracellular GSH levels, suggesting that interneurons contain a highly reducing environment that maintains HIF-1 stability and expression during ischemia.

\section{Materials and methods Isolation of neurons}

Primary neuronal cultures of cerebral cortices were obtained from Sprague-Dawley (SD) rat brains (postnatal day 0 [P0] to P3). Cultures were prepared according to Brewer et al. [15] with slight modifications. Whole cerebral cortices were dissected and then incubated for $50 \mathrm{~min}$ in $0.12 \%$ trypsin at $37^{\circ} \mathrm{C}$. After the incubation, cells were washed completely with Hank's balanced salt solution (HBSS) four times and dissociated with a firepolished glass pipette in dissociation medium (HBSS, $0.1 \% \mathrm{BSA}$ and $8 \mathrm{mM} \mathrm{MgCl}_{2}$ ), pelleted by centrifugation at $4000 \mathrm{~g}$ for $4 \mathrm{~min}$ at room temperature (RT), dissociated in starter medium (DMEM containing 10\% FCS) and plated on coverslips. Coverslips were pretreated by incubation with poly-D-lysine $(0.01 \%)$ for 1 hour (hr), then rinsed with sterile distilled water four times and dried before the cells were plated. Cultures were kept at $37^{\circ} \mathrm{C}$ in $5 \% \mathrm{CO}_{2}$ for $1 \mathrm{hr}$, flooded with starter medium and incubated overnight at $37^{\circ} \mathrm{C}$ in $5 \% \mathrm{CO}_{2}$. After $24 \mathrm{hrs}$, the medium was replaced with culture medium (Neurobasal plus $2 \mathrm{mM}$ glutamine and B27 supplement). Every 4-6 days, half of the medium was replaced with fresh culture medium. After 12-18 days of in vitro culture (DIV), neuronal cells were used for the experiments.

\section{In vitro hypoxia or cobalt chloride $\left(\mathrm{CoCl}_{2}\right)$ treatment}

Neuronal medium was removed and replaced with fresh, serum-free experimental medium (DMEM) containing 0, 5 , or $25 \mathrm{mM}$ glucose. For normoxia, neurons were incubated under $21 \%$ oxygen at $37^{\circ} \mathrm{C}$ for $3 \mathrm{hrs}$. For hypoxia, cells were maintained with $1 \% \mathrm{O}_{2} / 5 \% \mathrm{CO}_{2}$ balanced with $\mathrm{N}_{2}$ at $37^{\circ} \mathrm{C}$ for 3 or $5 \mathrm{hrs}$. Incubation periods were selected based on our previous studies [14]. Expression of HIF- $1 \alpha$ was observed in the cultured neurons after 3- and 5-hr hypoxic treatments, and further studies were performed only with 3-hr hypoxic treatments. For $\mathrm{CoCl}_{2}$ treatment, cortical neurons were incubated with $0.3 \mathrm{mM}$ $\mathrm{CoCl}_{2}$ at $37^{\circ} \mathrm{C}$ for $3 \mathrm{hrs}$ under normoxic conditions [16].

\section{Middle cerebral artery occlusion (MCAO)}

Male SD rats (Charles River Laboratories, Wilmington, MA, USA) weighing between $250 \mathrm{~g}$ and $280 \mathrm{~g}$ were used in accordance with the Guide for the Care and Use of Laboratory Animals and with approval from the Institutional Animal Care and Use Committee. Middle cerebral ische$\mathrm{mia}$ /reperfusion was conducted according to the method of Longa et al. (1989), with some modification. Briefly, the right common carotid artery, including its bifurcation, was dissected, and the external carotid artery was divided, leaving a stump of 3-4 $\mathrm{mm}$. The internal carotid artery was isolated, and the stump of the external carotid artery was reopened, and a 4.0 monofilament nylon suture with a slightly enlarged and rounded tip (Doccol Cooperation, Redlands, CA, USA) was inserted $17 \mathrm{~mm}$ through the internal carotid artery. Reperfusion was initiated by withdrawal of the filament at $90 \mathrm{~min}$ after occlusion. After surgery, the animals were allowed to recover from anesthesia while being given food and water ad libitum. Successful MCAO models were confirmed by 2, 3, 5 -triphenyltetrazolium chloride staining (Figure 1) and by behavioral observation according to Rogers' scales [17].

\section{In vitro immunocytochemistry}

Immunocytochemistry was performed as described by Ramamoorthy et al. [18]. Briefly, cortical neurons were washed with phosphate buffered saline (PBS) and fixed with $4 \%$ paraformaldehyde in PBS for $20 \mathrm{~min}$ at RT. Fixed cells were washed with PBS and permeabilized using $0.3 \%$ Triton X-100 in PBS for $15 \mathrm{~min}$ at RT and incubated with blocking solution (PBS containing 0.05\% Triton X-100 and $0.25 \%$ BSA) for $30 \mathrm{~min}$ at RT. Then, neurons were incubated with specific primary antibodies overnight at $4^{\circ} \mathrm{C}$. Cells were washed with blocking solution for $4 \times 15 \mathrm{~min}$ and incubated with an appropriate secondary antibody for $90 \mathrm{~min}$ at RT in the dark. Coverslips were washed with blocking solution and mounted with the temporary mounting medium Vectashield $\mathrm{H}$ 1000 (Vector Laboratories, Burlingame, CA). Fluorescent intensity was quantified with Image-Pro Plus 5.1 


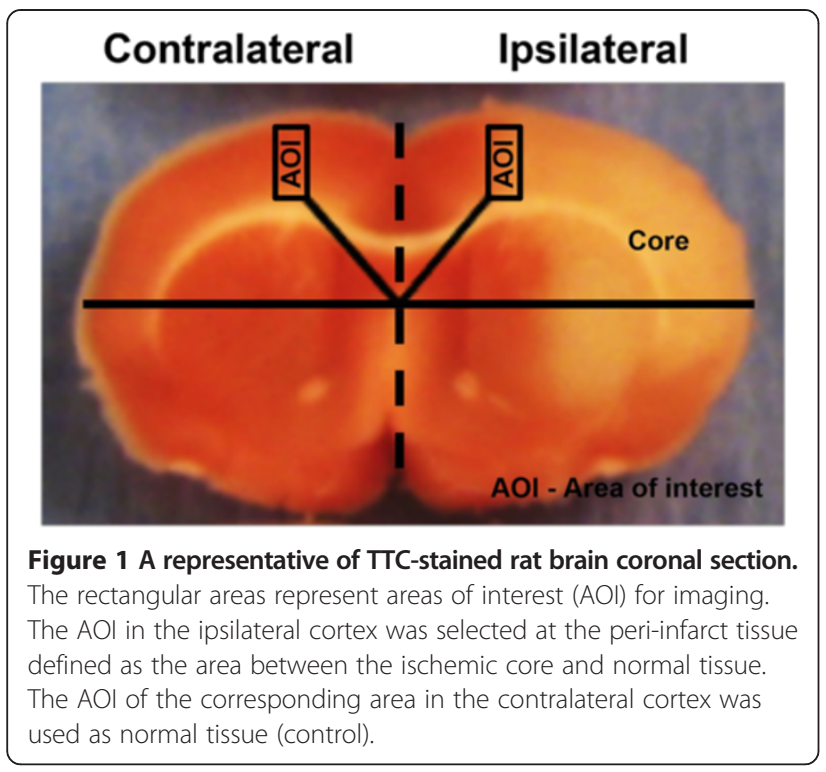

(Media Cybernetics). We randomly selected several cell free areas and calculated mean intensity as background intensity. We then determined fluorescent intensity in neuronal soma in normoxic and hypoxic conditions. The background intensity was subtracted from neuronal HIF- $1 \alpha$ intensity. Changes in HIF- $1 \alpha$ intensity caused by hypoxia were normalized to the normoxic level.

\section{In vivo immunocytochemistry}

Rat brain was dissected after $90 \mathrm{~min}$ MCAO and $24 \mathrm{hr}$ reperfusion. In vivo fixative was performed with $4 \%$ paraformaldehyde cardio perfusion. Then, brains were soaked in $4 \%$ paraformaldehyde overnight at RT and $30 \%$ sucrose for $48-72 \mathrm{hr}$ at $4^{\circ} \mathrm{C}$. Then, the brain was frozen using ice cold isopentane or liquid nitrogen for 2-3 min. Coronal brain sections were prepared from frozen brain sample using a cryostat. Slices were stained as described previously [18] with slight modifications. Briefly, after brain slices were washed with PBS and fixed (4\% paraformaldehyde in PBS at RT) for 90 min, fixative was removed, and slices were washed with PBS and permeabilized in $1 \%$ Triton X-100 in PBS overnight at RT. Slices were incubated in blocking solution (PBS containing $2 \% \mathrm{BSA}$ ) overnight at $4{ }^{\circ} \mathrm{C}$ and incubated for $48 \mathrm{hrs}$ at $4^{\circ} \mathrm{C}$ with a primary antibody. Slices were washed in blocking solution for $15 \mathrm{~min} \times 4$ times and incubated with a secondary antibody for $4 \mathrm{hrs}$ at RT. After washing, the slices were mounted in the temporary mounting medium Vectashield H1000. Control experiments were performed by omitting the primary antibody and incubating with secondary antibody alone.

Primary antibodies used were goat anti-HIF-1 $\alpha$ (1-100; sc-8711; Santa Cruz Biotechnology), rabbit anti-HIF-1 $\alpha$
(1-1000; 04-1006; Millipore Bioscience), mouse antiHIF-1 $\alpha$ (1-200; NB-100-123; Novus Biological), mouse anti-MAP2 (1:200; MAB378; Millipore Bioscience) and rabbit anti-GAD65/67 (1-400; AB1511; Millipore Bioscience). Secondary antibodies were donkey anti-goat Alexa 488 (1-100; Molecular Probes), goat anti-rabbit Alexa 488 (1-100; Molecular Probes), goat anti-mouseFITC (1-50; Santa Cruz), donkey anti-mouse TRITC (1-50; Jackson ImmunoResearch) and donkey anti-rabbit DyLight 549 (1-2,500; Rockland). For double-staining experiments, antibodies were applied sequentially, starting with the anti-HIF- $1 \alpha$ antibody. For antibody specificity, goat anti-HIF- $1 \alpha$ antibody was incubated with HIF- $1 \alpha$ blocking peptide (1-100; Santa Cruz) at RT for 2 hrs before staining the neurons for HIF-1 $\alpha$. Control experiments showed no significant bleed-through of the fluorescent labels or cross-reactivity between antibodies. As marked in Figure 1, the peri-infarct tissues in the ipsilateral cortex and the corresponding area in the contralateral cortex were selected as areas of interest. Images were obtained with a Leica DMI4000 microscope with a 40X objective and a Leica DFC340 FX Digital camera, using Leica LAS AF software. For quantification, the intensity of HIF- $1 \alpha$ in the neuronal soma was randomly counted using MAP2- or GAD65/67-positive cell bodies. Fluorescent intensity was determined as described above.

\section{GSH staining}

GSH was detected by using monochlorobimane (MCB) as reported by Chatterjee et al. [19]. Briefly, after hypoxic treatments, $0.1 \mathrm{mM}$ of $\mathrm{MCB}$ was added directly to the neuronal petri dish and incubated for $20 \mathrm{~min}$ at $37^{\circ} \mathrm{C}$. After the incubation, neurons were fixed and stained for specific neuronal markers using the method described above. The staining process was performed in the dark. MCB-GSH was visualized at an excitation wavelength of $380 \mathrm{~nm}$ and an emission wavelength of $480 \mathrm{~nm}$ using a fluorescence microscope. For quantification, MCB-GSH intensity was calculated for the neuronal soma. As it is impossible to measure MCB-GSH intensity in the same neurons before and after hypoxic treatments, different plates of neurons were treated with normoxia and hypoxia. Fluorescent intensity was determined as described above.

\section{Cell death}

Dead or unhealthy neurons were counted visually by assessing morphological damage with the cytoskeletal protein MAP2 [20]. Healthy cells were identified by intact somata and processes, unhealthy cells were differentiated by swelling or bulging in the MAP2-positive processes and dead neurons were identified by disruption/breakdown in the MAP2 staining in both the cell body and processes. 


\section{Statistical analysis}

Image-Pro Plus, OriginPro7, and Excel were used for data analysis. One-way ANOVA and Student's $t$-test were used for overall significance. Data are presented as the mean \pm SD from at least 3 separate experiments. Differences were considered significant at $\mathrm{p}<0.05$.

\section{Results}

HIF-1a stability after hypoxia/ischemia is neuron-typespecific

Earlier studies have demonstrated that hypoxia/ischemia induces HIF- $1 \alpha$ expression in cortical neurons. However, the cortical region of the brain contains multiple neuronal types with different survival rates during ischemia. We initially asked two questions. First, do all types of cortical neurons express HIF- $1 \alpha$ in ischemia? Second, do only the surviving neurons express HIF- $1 \alpha$ in ischemia? To address these questions, we examined the expression of HIF- $1 \alpha$ in cultured cortical neurons exposed to hypoxia/ischemia. Under normoxic conditions, both round-soma-like and pyramidal-like neurons showed intact somata and processes; as expected, no expression of HIF- $1 \alpha$ was seen (Figure 2A). Under hypoxic conditions, significant expression of HIF-1 $\alpha$ was only observed in certain MAP2-positive neurons. Interestingly, all the neurons that expressed HIF-1 $\alpha$ possessed round somata. This phenomenon was seen when cells were cultured in 5 or $25 \mathrm{mM}$ glucose under $1 \%$ oxygen (Figure $2 \mathrm{~A}^{\prime}$ ). The neurons with a pyramidal-like morphology showed no significant HIF-1 $\alpha$ expression and had swollen somata and bulging/broken processes in both 5 and $25 \mathrm{mM}$ glucose after hypoxia. Furthermore, when the neurons were treated with $0 \mathrm{mM}$ glucose for $3 \mathrm{hrs}$, almost all the MAP2-positive neurons showed swelling or breakdown in their processes (unhealthy/dead) under normoxic conditions (data not shown). For further confirmation of the high expression of HIF- $1 \alpha$ in the neurons with round somata, these experiments were repeated using cobalt chloride $\left(\mathrm{CoCl}_{2}\right)$ treatment, a HIF-1 $\alpha$ inducer. $\mathrm{CoCl}_{2}$-induced HIF-1 $\alpha$ expression was found in the
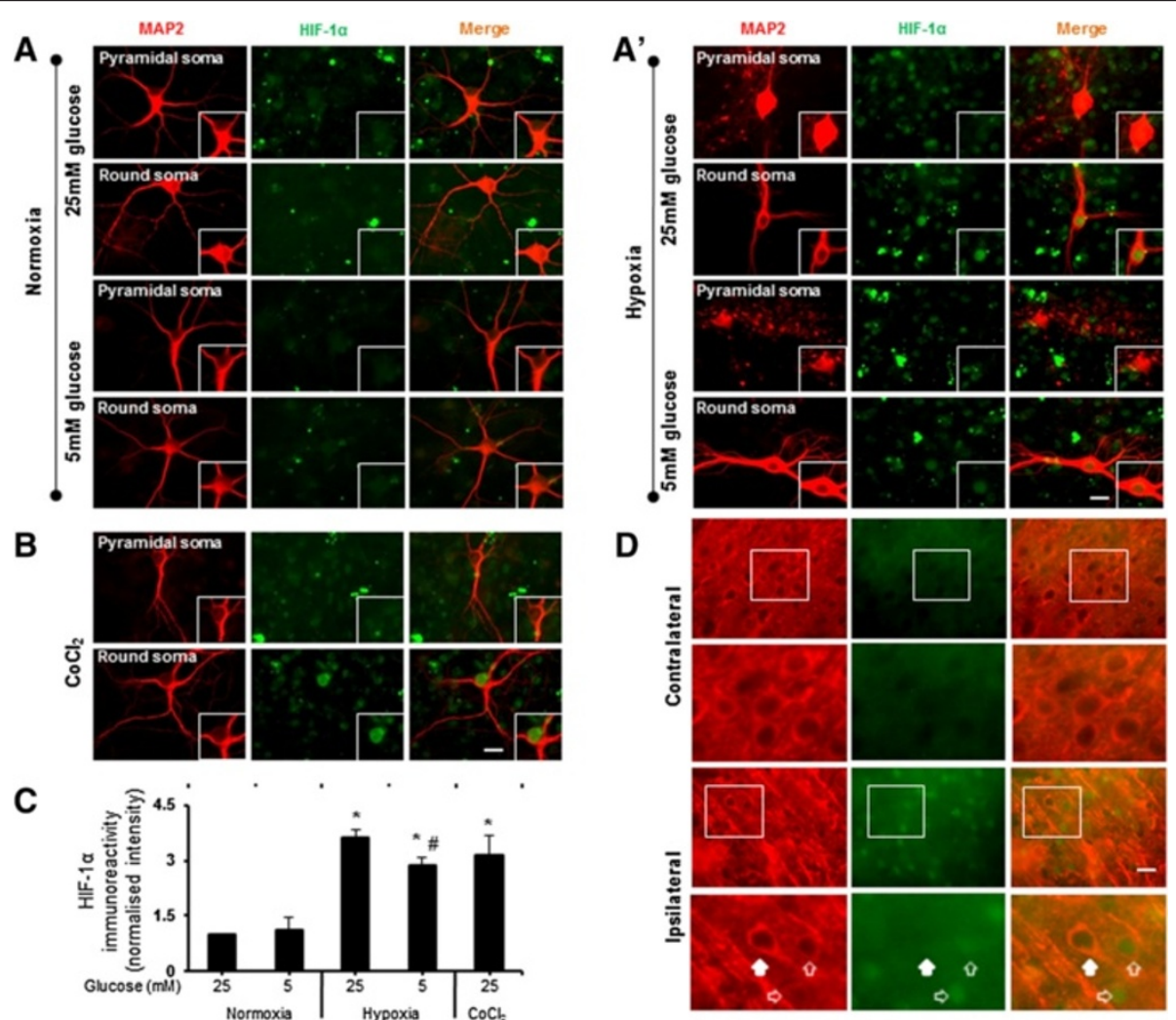

Figure 2 HIF-1a expression in primary cortical neurons exposed to hypoxia/ischemia. A \& A') Neurons were double-stained for HIF-1a and MAP2 in the presence of 5 and $25 \mathrm{mM}$ glucose with and without hypoxia. HIF-1a expression in the somata was observed in cells with interneuron-like morphology after hypoxia. B) $\mathrm{CoCl}_{2}(0.3 \mathrm{mM})$ induced HIF-1a expression in cells with interneuron-like morphology. C) Quantification represents the increase in HIF-1 a-ir staining (mean \pm SD; 5-10 neurons quantified from each experiment, $\mathrm{n}=3$ experiments). D) In vivo brain slice shows a similar pattern of positive HIF-1a -ir in round soma (open arrow) and negative in neurons with pyramidal-like morphology (solid arrow) in the ipsilateral side. ${ }^{*} \mathrm{p}<0.05$, compared with normoxia (25 mM glucose), \#p<0.05, compared with hypoxia (25 mM glucose). Scale bars, $20 \mu \mathrm{m}$ (A, A', B); $10 \mu \mathrm{m}$ (D). 
neurons that contained round somata (Figure 2B, bottom panel) but not those with pyramidal-like morphology (Figure 2B, upper panel). Analysis of the total intensity of HIF- $1 \alpha$-immunoreactive (ir) neurons with round somata showed 3.0- and 3.5-fold increases in response to $5 \mathrm{mM}$ and $25 \mathrm{mM}$ glucose under hypoxia, respectively (Figure $2 \mathrm{C}$ ).

To further confirm these findings, we examined HIF$1 \alpha$ expression in different neurons in the cortex of a rat MCAO model. Double staining of HIF- $1 \alpha$ and MAP2 in coronal brain slices revealed that not all MAP2-ir neurons expressed HIF- $1 \alpha$ in the cortical area. Similar to our observation in primary cultures, neurons with a round-soma-like morphology showed HIF-1 $\alpha$-ir in the ipsilateral region (open arrow), but no HIF- $1 \alpha$ staining was observed in the contralateral region (Figure 2D). In addition, neurons with a pyramidal-like morphology showed no HIF-1 $\alpha$ expression (Figure 2D solid arrow), confirming the in vitro data.

The above results demonstrated that the neuronal subpopulation containing round somata with intact cell bodies expressed HIF-1 $\alpha$ under hypoxia. To assess neuronal survival under these conditions, we quantified the neurons subjected to in vitro ischemia using MAP2-ir staining. Neurons were divided into three categories: 1) intact cell body and processes (viable cells), 2) swollen cell body with swollen processes (unhealthy or dead), and 3) disrupted cell body and broken processes (dead). Under normoxia, $80-90 \%$ of neurons were viable in the presence of either $5 \mathrm{mM}$ or $25 \mathrm{mM}$ glucose, but in the presence of $0 \mathrm{mM}$ glucose, the proportion of viable neurons dropped to $10 \%$. When exposed to $1 \%$ oxygen, $70-80 \%$ of neurons were unhealthy or dead after $3 \mathrm{hrs}$ in 5 or $25 \mathrm{mM}$ glucose concentrations. There was no significant difference between normoxia and hypoxia when the cells were exposed to $0 \mathrm{mM}$ glucose (Table 1). Further neuronal experiments were performed only with $25 \mathrm{mM}$ glucose.

Table 1 Hypoxia-induced neuronal viability in the presence of different glucose concentrations

\begin{tabular}{ll}
\hline Treatments & Viability (\%) \\
\hline Normoxia & \\
$25 \mathrm{mM}$ glucose & $90.18 \pm 3.00$ \\
$5 \mathrm{mM}$ glucose & $89.63 \pm 2.72$ \\
$0 \mathrm{mM}$ glucose & $10.43 \pm 5.02^{*}$ \\
Hypoxia & \\
$25 \mathrm{mM}$ glucose & $30.84 \pm 1.58^{*}$ \\
$5 \mathrm{mM}$ glucose & $22.27 \pm 5.37^{* \#}$ \\
$0 \mathrm{mM}$ glucose & $8.02 \pm 5.96^{* \#}$ \\
\hline
\end{tabular}

Cell viability was estimated by integrity of the soma and processes. ${ }^{*} \mathrm{p}<0.05$, compared to $25 \mathrm{mM}$ glucose, normoxia; ${ }^{\#} \mathrm{p}<0.05$, compared to $25 \mathrm{mM}$ glucose, hypoxia (mean $\pm \mathrm{SD} ; \mathrm{n}=4$ independent cultures).

\section{GAD65/67-positive neurons express HIF-1a following hypoxia/ischemia}

To confirm that the round-soma neurons that expressed HIF- $1 \alpha$ under hypoxia were GABAergic neurons, we double-stained neuronal cultures with antibodies against HIF-1 $\alpha$ and GAD65/67 (interneuronal marker). As shown in Figure 3, under normoxia, neurons did not express HIF-1 $\alpha$, regardless of their GAD65/67 expression. Some neurons showed co-localization of HIF- $1 \alpha$ and GAD65/67 under hypoxic conditions. No GAD65/67-negative cells were HIF-1 $\alpha$-ir whereas most GAD65/67-positive cells were HIF- $1 \alpha$-ir under hypoxia. We then quantified the percentage of HIF-1 $\alpha$-ir neurons showing coexpression of GAD65/67. These results demonstrated that $35-40 \%$ of GAD65/67-ir neurons were HIF-1 $\alpha$-ir (Figure 3B). For further confirmation, we co-stained brain slices from the rat model of MCAO with HIF-1 $\alpha$ and GAD65/67 antibodies. These data demonstrated that HIF-1 $\alpha$-ir neurons co-localized with GAD65/67-ir neurons (Figure 3C, 1). It is noteworthy that not all GAD65/67-ir neurons in the brain slices expressed HIF-1 $\alpha$ (Figure 3B, 2). These results confirmed that HIF- $1 \alpha$ expression was specific to interneurons and that not all cortical interneurons expressed HIF- $1 \alpha$ after ischemia.

\section{GSH stabilizes HIF-1a in interneurons after hypoxia/ischemia}

We have previously reported that redox status plays an important role in HIF- $1 \alpha$ expression in cortical neurons [14]. We postulated that GSH might play a role in regulating HIF-1 $\alpha$ expression in different types of neurons. We therefore used MCB staining to evaluate levels of GSH in pyramidal neurons and interneurons exposed to hypoxia. Figure 4A shows that MCB-GSH intensity was equally expressed in all MAP2-ir neurons under normoxia. Interestingly, neurons with pyramidal-like morphology had less MCB-GSH staining under hypoxia than under normoxia. Neurons with interneuron-like morphology showed higher MCB-GSH staining in MAP2-ir neurons than the neurons with pyramidal-like somata after exposure to hypoxia. To confirm whether this increase in MCB-GSH was co-localized with GAD65/67 neurons, we stained the neurons for GAD65/67 after MCB treatments with and without hypoxia. Some of the GAD65/67-ir neurons showed an increase in the MCB-GSH signal under hypoxia compared to normoxia (Figure $4 \mathrm{~B}$, open arrow). GAD65/67-negative neurons with pyramidal-like somata showed reduced MCB-GSH staining during hypoxia (Figure 4B, lower panel, solid arrow). The inset in Figure $4 \mathrm{~B}$ shows a phase-contrast image to confirm that the GAD65/67-negative neuron had a pyramidal-like soma. We then examined whether the neurons with an increase in MCB-GSH also expressed HIF-1 $\alpha$. This was done by co-staining the neurons with HIF-1 $\alpha$ and 


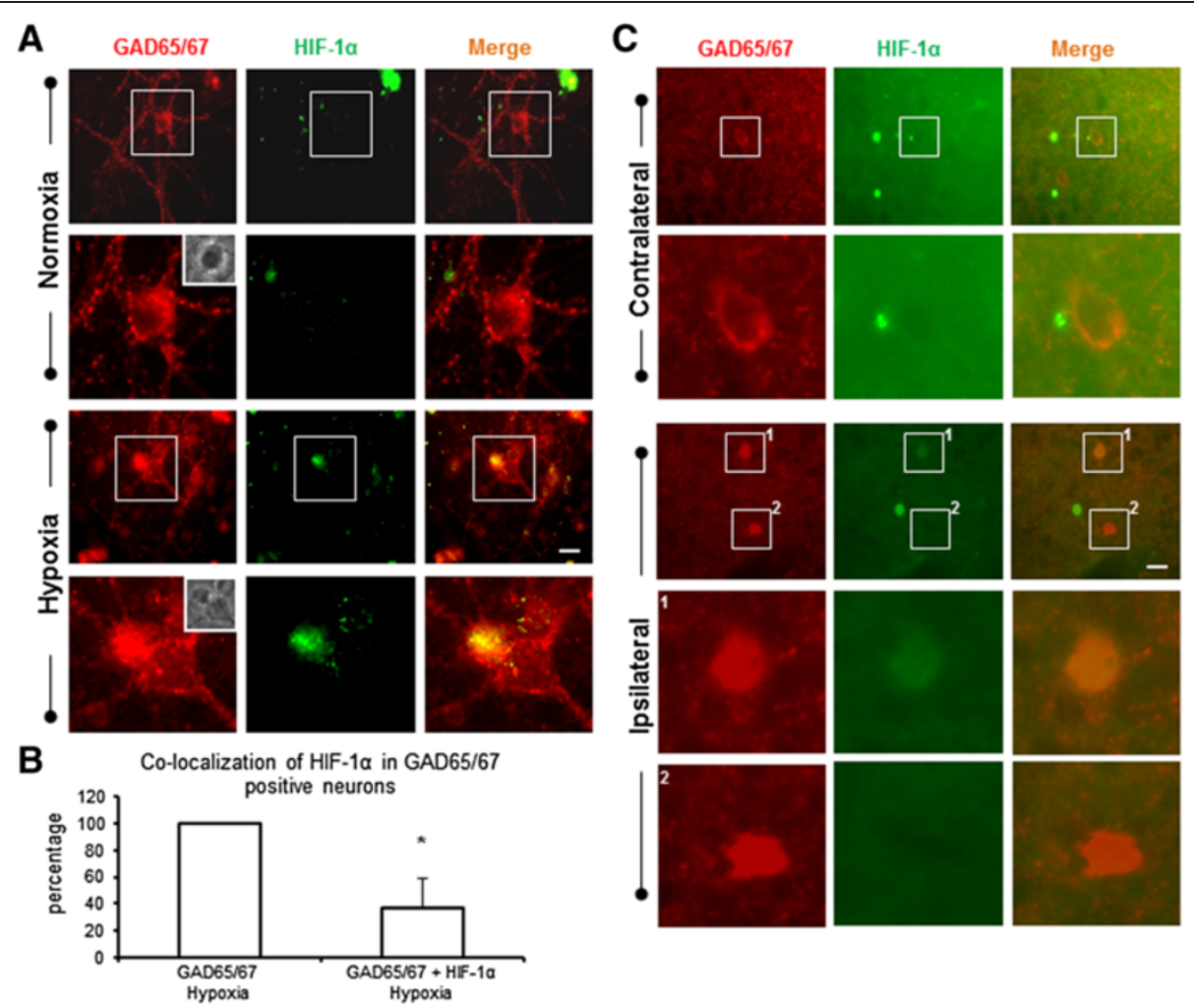

Figure 3 GAD65/67-positive neurons expressed HIF-1a under hypoxic conditions. A) HIF-1a expression co-localized with GAD65/67-ir in neurons exposed to hypoxia when compared to normoxia (upper panel) or GAD65/67-negative neurons in hypoxia (bottom panel, open arrow). B) Quantification shows the percentage of HIF-1a-expressing GAD65/67-positive neurons after hypoxia in vitro (mean \pm SD; from $n=6$ cultures). C) In vivo immunostaining illustrates HIF-1a-positive (bottom panel, solid arrow) and HIF-1a-negative (bottom panel, open arrow) in GAD65/67-ir neurons in the ipsilateral region, whereas the contralateral region shows no HIF-1a staining in GAD65/67-ir neurons (see Figure 1 for region selection). Scale bars, $10 \mu \mathrm{m}$ (A); $20 \mu \mathrm{m}$ (B).

GAD65/67 after MCB treatment. Normoxic neurons, including the GAD65/67-positive cells, did not show HIF-1 $\alpha$-ir, (Figure $4 \mathrm{C}$, upper panel). Following hypoxia, neurons showed HIF-1 $\alpha$ expression only in GAD65/67-ir neurons containing high levels of MCB-GSH (Figure 4C, middle panel and line profile). Interestingly, not all GAD65/67-ir neurons had increased levels of MCB-GSH under hypoxia. Quantification of the data revealed that almost 35-40\% of GAD65/67-ir neurons were HIF-1 $\alpha$-ir and possessed high levels of MCB-GSH (Figure 4D). Furthermore, inhibition of GSH synthesis by buthionine sulfoximine (BSO) reduced the overall MCB-GSH intensity and suppressed HIF-1 $\alpha$ expression in all GAD65/67-ir neurons exposed to hypoxia (Figure $4 \mathrm{C}$, bottom panel). Quantitative data indicated that when the MCB-GSH level was reduced to $30-40 \%$ with $\mathrm{BSO}$, the expression of HIF-1 $\alpha$ in GAD65/67 positive neurons was completely inhibited (Figure 4E). These results suggest that differences in the level of GSH may be responsible for different levels of HIF-1 $\alpha$ expression in hypoxic interneurons and pyramidal neurons.

\section{Discussion}

The present study demonstrates for the first time that HIF- $1 \alpha$ expression is cell-type-specific among cortical neurons in response to hypoxic/ischemic insults. Specifically, certain interneurons express a significantly higher level of HIF- $1 \alpha$ protein than pyramidal neurons. Furthermore, the present results reveal that reduction in the GSH level might play a role in decrease in the HIF- $1 \alpha$ level of the interneurons.

Previous reports have shown that the two major classes of neurotransmitter-containing neurons, pyramidal neurons and interneurons, are differentially affected during ischemia [3,21]. Consistent with these reports, our results demonstrate that hypoxia induces swelling in the somata or disruption of processes in pyramidal neurons. In contrast, the interneurons show intact somata and processes during hypoxia. Pyramidal neurons and interneurons have different mechanisms for buffering intracellular calcium during hypoxia [22,23]. Interneurons contain specific calcium-binding proteins (e.g., parvalbumin (PV) and calbindin $(\mathrm{CaBP})$ ) to regulate intracellular calcium for the 


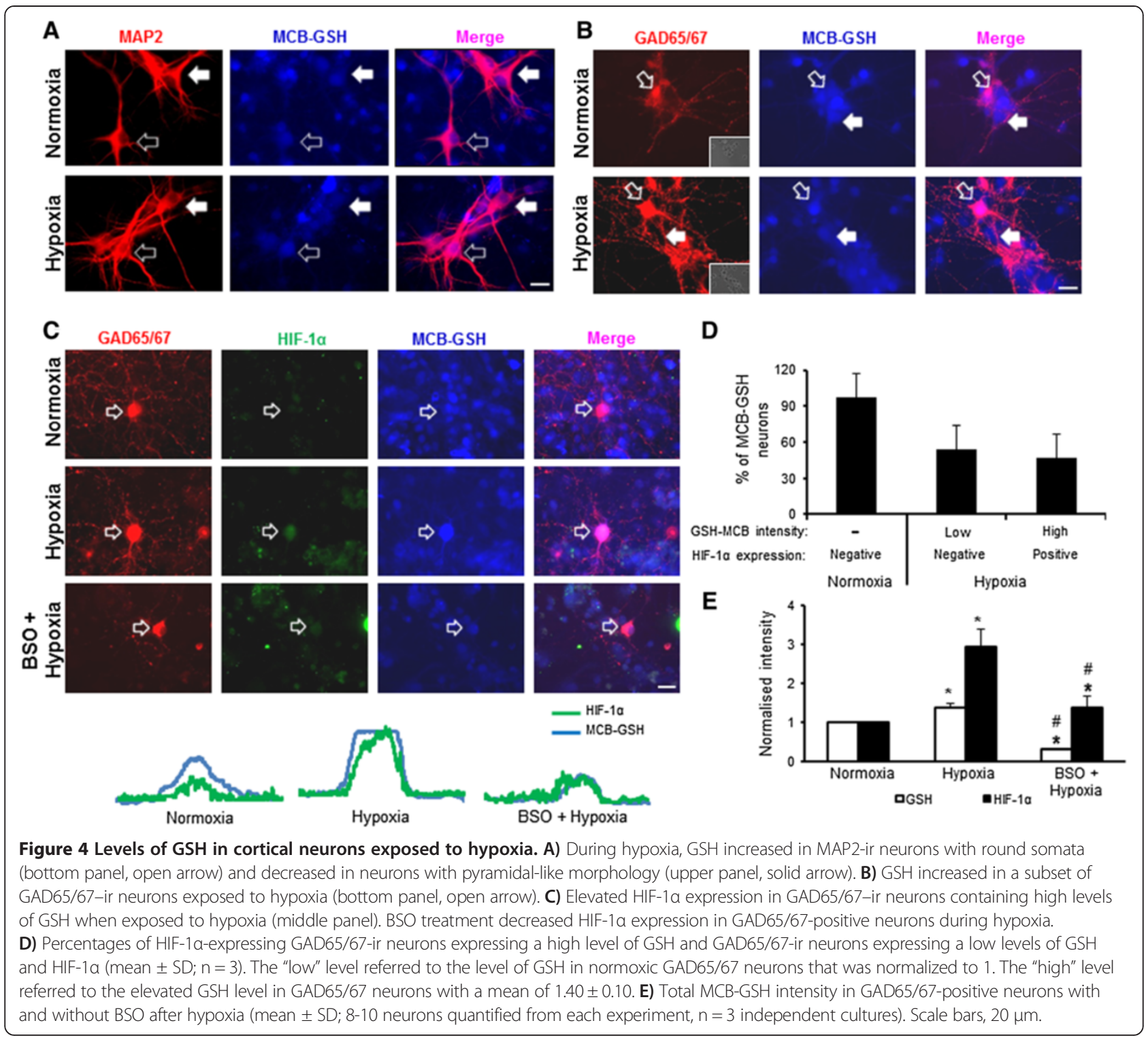

survival of these cells $[24,8]$. Because calcium is a critical damaging factor in ischemic neuronal death, these calcium-binding proteins might contribute to interneuronal survival. However, Freund et al. found that there was no consistent and systematic relationship between neuronal $\mathrm{CaBP}$ or PV content and ischemic vulnerability [25]. They and Crain et al. demonstrated that the majority of supragranular pyramidal cells that contained CaBP were the pyramidal cells most frequently degenerating after ischemia $[26,25]$. In contrast, the density and distribution of non-pyramidal cells, mainly interneurons, containing $\mathrm{CaBP}$ or $\mathrm{PV}$ appeared qualitatively unchanged after ischemia $[25,26]$. In addition, Larsson et al. have reported that increased neurotrophin signaling may provide neuroprotection in ischemic interneurons [27]. Our results clearly demonstrate that among the cortical neurons,
GAD65/67-positive neurons, but not pyramidal neurons, express HIF-1 $\alpha$ following hypoxia. These results indicate a novel pathway that may contribute to the resistance of interneurons to ischemia. Consistent with this concept, HIF-1 has been found to regulate transcription of the NTRK2 gene, which stimulates neurotrophin signaling [28].

It is worth noting that $\mathrm{CoCl}_{2}$ treatments also induce significant expression of HIF- $1 \alpha$ in interneurons, whereas minimal expression is seen in neurons containing pyramidal-like somata and apical dendrites with intact cell bodies and processes. The dose of $\mathrm{CoCl}_{2}$ used in the present experiments does not cause cell death in either interneurons or pyramidal neurons. This indicates that the expression of HIF- $1 \alpha$ in hypoxic interneurons is not necessarily a result of cell survival. 
Many factors can regulate HIF- $1 \alpha$ protein stability in an oxygen-independent manner. For example, heat shock protein 90 can stabilize HIF-1 $\alpha$, and receptor for activated kinase C (RACK1) can lead to HIF-1 $\alpha$ ubiquitination and degradation [29]. Calcium might regulate HIF-1 $\alpha$ expression in ischemic neurons. However, the available results are controversial or contradictory to each other. Calcineurin, a calcium-dependent protein phosphatase, may promote HIF-1 $\alpha$ expression by de-phosphorylating RACK1 [30]. In contrast, it has been reported that lowering the intracellular calcium concentration activates HIF-1 through inhibition of hydroxylation of HIF-1 $\alpha$ [31]. Unlike these results, Salnikow et al. reported that elevation of intracellular calcium neither induced the expression of HIF-1 $\alpha$ protein nor stimulated HIF-1-dependent transcription [32]. However, Mottet et al. argued that elevated calcium levels after prolonged hypoxia increased extracellular signal-regulated kinase $1 / 2$ (ERK 1/2) activation and increased HIF-1 transcriptional activity but did not induce HIF- $1 \alpha$ accumulation [33]. Our results clearly show that GSH can stabilize HIF- $1 \alpha$ protein in ischemic neurons. This is consistent with previous reports that HIF- $1 \alpha$ can be strongly regulated by the redox environment [34-36], a mechanism that is of particular importance in the setting of ischemic brain injury because of the intrinsic changes in redox status. Although a more oxidizing environment has been suggested to stabilize HIF-1 $\alpha$ in non-neuronal cells $[37,38]$, there is strong evidence supporting that a more reducing environment stabilizes HIF-1 $\alpha$, such as in COS7 cells [39], HeLa cells [40], HepG2 cells [41], MCF-7 cells [34], salmonid cells [42], renal medullary interstitial cells [43], and primary cultured neurons [14]. Reactive oxygen species can increase the activity of both $26 \mathrm{~S}$ and $20 \mathrm{~S}$ proteasomal degradation pathways under hypoxic conditions $[44,45]$. Thus, it is highly possible that the presence of high levels of GSH decreases the activity of the 20S and 26S pathways and stabilizes HIF- $1 \alpha$ protein in interneurons. Based on this discussion, GSH may not be the only factor contributing to HIF- $1 \alpha$ expression in the two neuronal types. Other antioxidants that are able to reduce reactive oxygen species in the neurons may also contribute to the stabilization of HIF- $1 \alpha$ protein.

The exact mechanism by which hypoxic interneurons maintain higher levels of GSH than hypoxic pyramidal neurons remains unclear. However, the following mechanisms may be involved. First, interneurons may generate lower amounts of free radicals than pyramidal neurons in hypoxia/ischemia. It is well established that ischemia causes excess free radical generation, mainly via mitochondrial dysfunction caused by excessive calcium. Interneurons can have lower levels of NMDA receptor activity by inhibiting Cys-299 of the NMDA receptor subunit NR2A [46]. This inactivation of glutamate receptors reduces the intracellular calcium and thus reduces free radical generation. Second, interneurons may have an enhanced defense system against oxidative stress. Interneurons have an enhanced and more efficient thioredoxin-2 system in detoxifying hydrogen peroxide, compared to pyramidal neurons [47]. Enhanced expression of antioxidants such as superoxide dismutase and Bcl-2 has been observed in ischemic interneurons [46]. In addition, the presence of nitric oxide may also help interneurons reduce free radical generation and protease activity [48,46]. Overall, both low levels of free radical generation and enhanced levels of antioxidants may spare GSH in hypoxic interneurons.

\section{Conclusion}

We report here that expression of HIF- $1 \alpha$ is mainly detected in interneurons during hypoxia/ischemia. High levels of GSH in interneurons may play a role in maintaining the cellular environment for the expression of HIF-1 $\alpha$. These results provide essential information for understanding the pathophysiology of cerebral ischemia.

\section{Abbreviations}

CaBP: Calbindin; GABA: Y-aminobutyric acid; GSH: Glutathione; HIF-1: Hypoxia inducible factor-1; HBSS: Hank's balanced salt solution; MCAO: Middle cerebral artery occlusion; MCB: Monochlorobimane; PHD: Proline hydroxylase; RT: Room temperature.

\section{Competing interests}

The authors declare that they have no competing interests.

\section{Authors' contributions}

PR carried out experiments, participated in experimental design and data analyses, and drafted the manuscript. HS participated in the design of the study, data analyses, and finalizing the manuscript. Both authors read and approved the final manuscript.

\section{Authors' information}

PR is an assistant research professor at University of Kansas Medical Center. $\mathrm{HS}$ is an associate professor in pharmacology, toxicology and neuroscience at School of Pharmacy, University of Kansas.

\section{Acknowledgments}

This research was supported in part by a grant from the National Institutes of Health (R01NS058807) and a Kansas University Center for Research startup fund.

Received: 18 March 2014 Accepted: 29 April 2014

Published: 5 May 2014

\section{References}

1. Monnerie $H$, Le Roux PD: Reduced dendrite growth and altered glutamic acid decarboxylase (GAD) 65- and 67-kDa isoform protein expression from mouse cortical GABAergic neurons following excitotoxic injury in vitro. Exp Neurol 2007, 205(2):367-382. doi:S0014-4886(07)00082-9.

2. Chesselet MF, Gonzales C, Lin CS, Polsky K, Jin BK: Ischemic damage in the striatum of adult gerbils: relative sparing of somatostatinergic and cholinergic interneurons contrasts with loss of efferent neurons. Exp Neurol 1990, 110(2):209-218. doi:0014-4886(90)90032-N.

3. Frahm C, Haupt C, Witte OW: GABA neurons survive focal ischemic injury. Neuroscience 2004, 127(2):341-346. doi:10.1016/j.neuroscience.2004.05.027.

4. Katchanov J, Waeber C, Gertz K, Gietz A, Winter B, Bruck W, Dirnagl U, Veh RW, Endres M: Selective neuronal vulnerability following mild focal brain ischemia in the mouse. Brain Pathol 2003, 13(4):452-464. 
5. Aarts M, Liu Y, Liu L, Besshoh S, Arundine M, Gurd JW, Wang YT, Salter MW, Tymianski M: Treatment of ischemic brain damage by perturbing NMDA receptor- PSD-95 protein interactions. Science 2002, 298(5594):846-850. doi:10.1126/science.1072873.

6. Fryd Johansen F, Balslev Jorgensen M, Diemer NH: Resistance of hippocampal CA-1 interneurons to 20 min of transient cerebral ischemia in the rat. Acta Neuropathol 1983, 61(2):135-140.

7. Johansen FF, Lin CT, Schousboe A, Wu JY: Immunocytochemical investigation of L-glutamic acid decarboxylase in the rat hippocampal formation: the influence of transient cerebral ischemia. J Comp Neurol 1989, 281(1):40-53. doi:10.1002/cne.902810105.

8. Ferrer I, Soriano MA, Vidal A, Planas AM: Survival of parvalbuminimmunoreactive neurons in the gerbil hippocampus following transient forebrain ischemia does not depend on HSP-70 protein induction. Brain Res 1995, 692(1-2):41-46. doi:0006-8993(95)00527-W

9. Giaccia A, Siim BG, Johnson RS: HIF-1 as a target for drug development. Nat Rev Drug Discov 2003, 2(10):803-811.

10. Baranova O, Miranda LF, Pichiule P, Dragatsis I, Johnson RS, Chavez JC Neuron-specific inactivation of the hypoxia inducible factor 1 alpha increases brain injury in a mouse model of transient focal cerebral ischemia. J Neurosci 2007, 27(23):6320-6332. doi:27/23/6320.

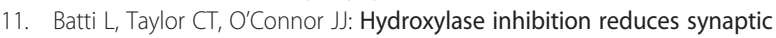
transmission and protects against a glutamate-induced ischemia in the CA1 region of the rat hippocampus. Neuroscience 2010, 167(4):1014-1024. doi:10.1016/j.neuroscience.2010.03.011

12. Vordermark D, Katzer A, Baier K, Kraft P, Flentje M: Cell type-specific association of hypoxia-inducible factor-1 alpha protein accumulation and radiobiologic tumor hypoxia. Int J Radiat Oncol Biol Phys 2004, 58(4):1242-1250. doi:10.1016/j.jijrobp.2003.11.030.

13. Zheng $X$, Ruas $J L$, Cao R, Salomons FA, Cao Y, Poellinger $L$, Pereira $T$ : Cell-type-specific regulation of degradation of hypoxia-inducible factor 1 alpha: role of subcellular compartmentalization. Mol Cell Biol 2006 26(12):4628-4641. doi:26/12/4628.

14. Guo S, Bragina $O, X u Y$, , Cao Z, Chen $H$, Zhou B, Morgan M, Lin Y, Jiang BH, Liu KJ, Shi H: Glucose up-regulates HIF-1alpha expression in primary cortical neurons in response to hypoxia through maintaining cellular redox status. J Neurochem 2008, 105(5):1849-1860. doi:JNC5287.

15. Brewer GJ: Serum-free B27/neurobasal medium supports differentiated growth of neurons from the striatum, substantia nigra, septum, cerebral cortex, cerebellum, and dentate gyrus. J Neurosci Res 1995, 42(5):674-683.

16. Tan XL, Huang XY, Gao WX, Zai Y, Huang QY, Luo YJ, Gao YQ: $\mathrm{CoCl}_{2^{-}}$ induced expression of p300 promotes neuronal-like PC12 cell damage. Neurosci Lett 2008, 441(3):272-276. doi:S0304-3940(08)00894-X

17. Rogers DC, Campbell CA, Stretton JL, Mackay KB: Correlation between motor impairment and infarct volume after permanent and transient middle cerebral artery occlusion in the rat. Stroke 1997, 28(10):2060-2065.

18. Ramamoorthy P, Whim MD: Trafficking and fusion of neuropeptide Y-containing dense-core granules in astrocytes. J Neurosci 2008, 28(51):13815-13827. doi:28/51/13815.

19. Chatterjee S, Noack H, Possel H, Keilhoff G, Wolf G: Glutathione levels in primary glial cultures: monochlorobimane provides evidence of cell type-specific distribution. Glia 1999, 27(2):152-161.

20. Cao F, Hata R, Zhu P, Takeda S, Yoshida T, Hakuba N, Sakanaka M, Gyo K: Delayed neuronal cell death in brainstem after transient brainstem ischemia in gerbils. BMC Neurosci, 11:115. doi:1471-2202-11-115.

21. Schlander M, Hoyer S, Frotscher M: Glutamate decarboxylaseimmunoreactive neurons in the aging rat hippocampus are more resistant to ischemia than CA1 pyramidal cells. Neurosci Lett 1988, 91(3):241-246

22. Pisani A, Calabresi P, Tozzi A, Bernardi G, Knopfel T: Early sodium elevations induced by combined oxygen and glucose deprivation in pyramidal cortical neurons. Eur J Neurosci 1998, 10(11):3572-3574.

23. Pisani $A$, Bonsi $P$, Calabresi $P$ : Calcium signaling and neuronal vulnerability to ischemia in the striatum. Cell Calcium 2004, 36(3-4):277-284 doi:10.1016/j.ceca.2004.02.010.

24. Freimann FB, Crome O, Shevtsova Z, Bahr M, Kugler S: Evaluation of long-term upregulation of Calbindin D28K as a preventive approach for ischaemic stroke. Int J Stroke, 5(4):319-320. doi:IJS446.

25. Freund TF, Buzsaki G, Leon A, Baimbridge KG, Somogyi P: Relationship of neuronal vulnerability and calcium binding protein immunoreactivity in ischemia. Exp Brain Res 1990, 83(1):55-66.
26. Crain BJ, Westerkam WD, Harrison AH, Nadler JV: Selective neuronal death after transient forebrain ischemia in the Mongolian gerbil: a silver impregnation study. Neuroscience 1988, 27(2):387-402.

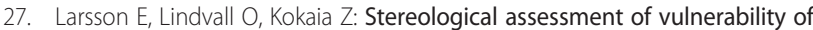
immunocytochemically identified striatal and hippocampal neurons after global cerebral ischemia in rats. Brain Res 2001, 913(2):117-132. doi:S0006-8993(01)02762-7.

28. Martens LK, Kirschner KM, Warnecke C, Scholz H: Hypoxia-inducible factor-1 (HIF-1) is a transcriptional activator of the TrkB neurotrophin receptor gene. J Biol Chem 2007, 282(19):14379-14388. doi:10.1074/jbc. M609857200.

29. Liu YV, Baek JH, Zhang H, Diez R, Cole RN, Semenza GL: RACK1 competes with HSP90 for binding to HIF-1alpha and is required for $\mathrm{O}(2)$-independent and HSP90 inhibitor-induced degradation of HIF-1alpha. Mol Cell 2007, 25(2):207-217. doi:10.1016/j.molcel.2007.01.001.

30. Liu YV, Hubbi ME, Pan F, McDonald KR, Mansharamani M, Cole RN, Liu JO, Semenza GL: Calcineurin promotes hypoxia-inducible factor 1alpha expression by dephosphorylating RACK1 and blocking RACK1 dimerization. J Biol Chem 2007, 282(51):37064-37073. doi:M705015200.

31. Berchner-Pfannschmidt U, Petrat F, Doege $K$, Trinidad B, Freitag P, Metzen E, de Groot H, Fandrey J: Chelation of cellular calcium modulates hypoxiainducible gene expression through activation of hypoxia-inducible factor-1alpha. J Biol Chem 2004, 279(43):44976-44986. doi:10.1074/jbc M313995200.

32. Salnikow $K$, Kluz T, Costa M, Piquemal D, Demidenko ZN, Xie K, Blagosklonny MV: The regulation of hypoxic genes by calcium involves c-Jun/AP-1, which cooperates with hypoxia-inducible factor 1 in response to hypoxia. Mol Cell Biol 2002, 22(6):1734-1741.

33. Mottet D, Michel G, Renard P, Ninane N, Raes M, Michiels C: Role of ERK and calcium in the hypoxia-induced activation of HIF-1. J Cell Physiol 2003, 194(1):30-44.

34. Welsh SJ, Bellamy WT, Briehl MM, Powis G: The redox protein thioredoxin-1 (Trx-1) increases hypoxia-inducible factor 1alpha protein expression: Trx-1 overexpression results in increased vascular endothelial growth factor production and enhanced tumor angiogenesis. Cancer Res 2002, 62(17):5089-5095.

35. Salceda S, Caro J: Hypoxia-inducible factor 1alpha protein is rapidly degraded by the ubiquitin-proteasome system under normoxic conditions. Its stabilization by hypoxia depends on redox-induced changes. J Biol Chem 1997, 272(36):22642-22647.

36. Wang $G L$, Jiang $B H$, Semenza GL: Effect of altered redox states on expression and DNA-binding activity of hypoxia-inducible factor 1 . Biochem Biophys Res Comm 1995, 212(2):550-556.

37. Chandel NS, Maltepe E, Goldwasser E, Mathieu CE, Simon MC, Schumacker PT: Mitochondrial reactive oxygen species trigger hypoxia-induced transcription. Proc Natl Acad Sci U S A 1998, 95(20):11715-11720.

38. Chandel NS, McClintock DS, Feliciano CE, Wood TM, Melendez JA Rodriguez AM, Schumacker PT: Reactive oxygen species generated at mitochondrial complex III stabilize hypoxia-inducible factor-1alpha during hypoxia: a mechanism of $\mathrm{O}_{2}$ sensing. J Biol Chem 2000, 275(33):25130-25138.

39. Carrero P, Okamoto K, Coumailleau P, O'Brien S, Tanaka H, Poellinger L: Redox-regulated recruitment of the transcriptional coactivators CREB-binding protein and SRC-1 to hypoxia-inducible factor 1alpha. Mol Cell Biol 2000, 20(1):402-415.

40. Huang LE, Arany Z, Livingston DM, Bunn HF: Activation of hypoxiainducible transcription factor depends primarily upon redox-sensitive stabilization of its alpha subunit. J Biol Chem 1996, 271(50):32253-32259.

41. Liu Q, Berchner-Pfannschmidt U, Moller U, Brecht M, Wotzlaw C, Acker H, Jungermann K, Kietzmann T: A Fenton reaction at the endoplasmic reticulum is involved in the redox control of hypoxia-inducible gene expression. Proc Natl Acad Sci U S A 2004, 101(12):4302-4307.

42. Nikinmaa M, Pursiheimo S, Soitamo AJ: Redox state regulates HIF-1alpha and its DNA binding and phosphorylation in salmonid cells. J Cell Sci 2004, 117(Pt 15):3201-3206.

43. Yang ZZ, Zhang AY, Yi FX, Li PL, Zou AP: Redox regulation of HIF-1alpha levels and HO-1 expression in renal medullary interstitial cells. Am J Physiol Renal Fluid Electr Physiol 2003, 284(6):F1207-F1215.

44. Shi $\mathrm{H}$ : Hypoxia inducible factor 1 as a therapeutic target in ischemic stroke. Curr Med Chem 2009, 16(34):4593-4600. doi:CMC - AbsEpub - 069. 
45. Kong X, Alvarez-Castelao B, Lin Z, Castano JG, Caro J: Constitutive/hypoxic degradation of HIF-alpha proteins by the proteasome is independent of von Hippel Lindau Protein Ubiquitylation and the transactivation activity of the protein. J Biol Chem 2007, 282(21):15498-15505.

46. Bidmon HJ, Emde B, Kowalski T, Schmitt M, Mayer B, Kato K, Asayama K, Witte OW, Zilles K: Nitric oxide synthase-I containing cortical interneurons co-express antioxidative enzymes and anti-apoptotic BCl-2 following focal ischemia: evidence for direct and indirect mechanisms towards their resistance to neuropathology. J Chem Neuroanat 2001, 22(3):167-184. doi:S0891-0618(01)00126-0.

47. Kudin AP, Augustynek B, Lehmann AK, Kovacs R, Kunz WS: The contribution of thioredoxin-2 reductase and glutathione peroxidase to $\mathrm{H}_{2} \mathrm{O}_{2}$ detoxification of rat brain mitochondria. Biochim Biophys Acta 2012, 1817(10):1901-1906. doi:10.1016/j.bbabio.2012.02.023.

48. Chiueh CC, Rauhala P: The redox pathway of S-nitrosoglutathione, glutathione and nitric oxide in cell to neuron communications. Free Radic Res 1999, 31(6):641-650.

doi:10.1186/2051-5960-2-51

Cite this article as: Ramamoorthy and Shi: Ischemia induces different levels of hypoxia inducible factor-1a protein expression in interneurons and pyramidal neurons. Acta Neuropathologica Communications 2014 2:51.

\section{Submit your next manuscript to BioMed Central and take full advantage of:}

- Convenient online submission

- Thorough peer review

- No space constraints or color figure charges

- Immediate publication on acceptance

- Inclusion in PubMed, CAS, Scopus and Google Scholar

- Research which is freely available for redistribution 